\title{
Psicogénesis del maltrato: una perspectiva psicoanalítica
}

\section{Psychogenesis of abuse: a psychoanalytic perspective}

Recibido: 6 de marzo 2021, evaluado: 13 de marzo 2021, aceptado: 7 de abril 2021

\author{
Orlando Cerna Dorregaray \\ https://orcid.org/0000-0002-1628-244X \\ ocerna@umch.edu.pe \\ Universidad Marcelino Champagnat
}

\section{Resumen}

El presente artículo estudia el maltrato desde la propuesta teórica psicoanalítica, para comprender sus causas y prevenirlo. Revisamos los estudios de Ferenczi en su famoso escrito: “Confusión de lengua entre los adultos y el niño". En este documento se postula que el maltrato ocurriría por un tipo de sumisión del niño hacia al agresor. Se desarrolla el concepto de identificación con el agresor y la introyección de lo que amenaza o agrede. Conceptos todos que son desarrollados en este artículo. También se revisan algunos estudios de psicoanalistas actuales que hacen referencia a los trabajos de Ferenczi y Freud. Todos contribuyen a entender los factores causales del maltrato o abuso. Adicionalmente, incluimos la reflexión sobre las causas de varios tipos de maltrato, tales como el físico, emocional, sexual y de género. Todo con la seguridad de que si comprendemos el maltrato desde su origen y conocemos sus causas, entonces podremos afrontarlo en nuestro entorno y sociedad en general.

Palabras claves: maltrato, identificación con el agresor, introyección de la culpa, dominio, sumisión, apego inseguro, ideales de género.

\section{Summary}

This article studies abuse from the theoretical psychonal proposal, to understand its causes and prevent it. We review Ferenczi's studies in his famous writing: "Confusion of language between adults and children." In this document it is postulated that the abuse would occur through a 
type of submission of the child to the aggressor. The concept of identification with the aggressor and the introjection of what is threatening or attacking is developed. All concepts that are developed in this article. Some studies of current psychoanalysts that refer to the works of Ferenczi and Freud are also reviewed. All contribute to understanding the causal factors of mistreatment or abuse. Additionally, we include reflection on the causes of various types of abuse, such as physical, emotional, sexual and gender. All with the assurance that if we understand abuse from its origin and know its causes, then we can confront it in our environment and society in general.

Keywords: abuse, identification with the aggressor, introjection of guilt, dominance, submission, insecure attachment, gender ideals.

\section{Introducción}

Frente a la anomia social que vivimos con relación al maltrato, nos proponemos en el presente escrito comprender el origen del mismo. Para ello presentamos la propuesta psicoanalítica que explica cómo se estructura el maltrato. Examinamos primero la realidad actual, la preocupación para entender esta problemática y planteamos una propuesta teórica para su comprensión y desde allí prevenir el maltrato de todo tipo.

La evidencia clínica de los psicoterapeutas sobre el maltrato en la infancia y la niñez es elevada. Sin embargo, también es evidente que existen una cantidad de adultos que han sido maltratados y no han llegado a la atención clínica psicoterapéutica. Esto se puede deducir de los constantes casos de maltrato que observamos en nuestro entorno. Es decir hay una población de adultos, no atendidos clínicamente y que son potenciales víctimas o maltratadores. No es posible sin ayuda profesional, psicoterapéutica, entender el maltrato, procesarlo y transformar ese suceso en una conducta por ejemplo resiliente. Pasar de maltratado/a a protector, a tutor de resiliencia en los términos de Cyrlunik (2010).

\section{Datos sobre el maltrato}

Presentamos a continuación algunos datos a nivel mundial sobre la situación de la violencia contra los menores. La OMS reporta diferentes datos y tipos de maltrato. Informa que el 
maltrato físico se da en un $23 \%$, el maltrato por negligencia o abandono en un $16 \%$, el maltrato emocional $36 \%$ y el abuso sexual $18 \%$ en niñas y $8 \%$ en niños. Estos datos de la OMS y tienen en cuenta la población infantil mundial.

Por otro lado, se informa que la mitad de los niños del mundo, unos mil millones de niños, y que se encuentran entre las edades de 2 a 17 años, se ven sujetos cada año a violencia física, sexual o psicológica, y sufren traumatismos, discapacidad y muerte.

Los datos del informe que estamos presentando se recopilaron en una encuesta que se llevó a cabo entre 2018 y 2019 a la que respondieron más de 1000 responsables de adoptar decisiones en 155 países. La violencia contra los niños incluye todas las formas de violencia contra los menores de 18 años, infligida por sus padres o por otras personas que les cuiden, sus compañeros, sus parejas u otras personas.

Se sabe además que 3 de 4 niños, que son 300 millones de niños, en edades entre 2 y 4 años sufren regularmente castigo físico o violencia psicológica de manos de sus padres o cuidadores. Por otro lado, 1 de 4 niños, que tienen menos de 5 años, viven con una madre que es víctima de violencia por parte de su pareja.

Así mismo 1 de 3 estudiantes, entre 13 y 15 años fueron maltratados físicamente el año pasado. Fueron $45 \%$ varones y $25 \%$ mujeres.

Otro dato importante es que 1 de 3 estudiantes, entre 11 y 15 años, sufrieron acoso escolar (bullying) el mes pasado. Los porcentajes de varones fueron de $35 \%$ varones y $30 \%$ de mujeres.

También se sabe que se han cometido 40,150 homicidios por año en niños cuyas edades van entre 0 a 17 años. Las cifras alcanzan a 28,160 varones y 11,990 mujeres.

Además se sabe que los niños entre 11 y 15 años que sufrieron de acoso escolar (bullying) tienen un $13 \%$ menos de probabilidades de concluir sus estudios secundarios.

Por otro lado, los adultos que experimentaron 4 o más adversidades en la niñez tienen un $7 \%$ más de probabilidades de verse involucrados en violencia interpersonal como víctimas o victimarios. Al mismo tiempo, estos adultos tienen un $30 \%$ más de probabilidades de intento de suicidio.

Finalmente, otro dato preocupante, es el que indica que los adultos varones que fueron abusados física o sexualmente de niños, tienen $14 \%$ más de probabilidades de perpetrar 
violencia física o sexual a su pareja. En el caso de las mujeres adultas, tienen un $16 \%$ más de probabilidades de sufrir de violencia física o sexual por parte de su pareja.

\section{Claves para entender el maltrato}

Las evidencia clínica de los psicoterapeutas que tratan estos casos, han señalado que los niños que son atendidos, registran que fueron maltratados entre los 2,3 y 4 años de edad. También se reporta que cuando estos niños maltratados llegan a la vida escolar y se producen actos de violencia entre pares, se reactivan en la memoria estos recuerdos. Por otro lado, estos niños evidencian un interés especial en los juegos violentos, les atraen y encuentran placer en ellos.

A continuación exponemos algunas claves que nos ayudarán a entender el maltrato.

\section{Claves sobre el maltrato en general:}

$1^{\circ}$ Hay más niños castigados físicamente.

$2^{\circ}$ Hay un reconocimiento que muchos niños no han sido castigados físicamente, pero han tenido un cuidador con mucha autoridad a su alrededor.

$3^{\circ}$ Algunos niños han fantaseado, imaginado que han sido maltratados, solo por lo que vivieron o escucharon de sus cuidadores; amenazas, agresiones hacia otros, etc.

$4^{\circ}$ Algunos niños sentían que los castigaban porque los querían o era el único momento donde les prestaban atención.

Estas claves se constatan cuando los datos o estadísticas informan que son más varones adultos los que maltratan físicamente a los menores, como una compulsión a la repetición de lo que a ellos les sucedió. Por otro lado, no solamente se trata de una repetición del maltrato, sino de haber vivido en un contexto autoritario, con cuidadores que instauraron el miedo, el temor en el niño que podría ser maltratado. Han existido casos reportados que asocian el maltrato con el único momento en que se les prestaba atención a los niños y también se ha reportado que era normal que al ser castigado les dijeran a algunos de estos niños, que lo hacían porque los querían. Como ese dicho: "te pego por tu bien" o "te pego porque te quiero, porque me importas" o el dicho: "pégale a tu hijo, de vez en cuando, aunque tú no sepas por qué lo haces; él sí sabrá por qué le estás pegando”. Estas frases han normalizado la violencia y el maltrato, incluso lo han asociado a un sentimiento de atención, de cariño, de amor por el otro. De este 
modo, podemos explicar muchos comportamientos violentos que en la actualidad se siguen dando, como si fueran normalizados o naturales.

\section{Claves sobre el maltrato a niñas:}

1. El castigo físico al niño significa una destitución del amor y humillación.

2. El castigo físico a la niña significa una atención, una forma de amor, del padre autoritario.

Veamos una pequeña viñeta de ejemplo. Una niña de 16 años, ante la muerte de su padre, dice: “Quisiera que estuviera vivo, aunque sea para pegarme". Ella sentía que su padre la amaba en ese instante. La niña de 16, luego se enamora y busca un pegador, no quiere un pegador, pero necesita un pegador. Esto se repite compulsivamente y el maltrato se instaura en las relaciones amorosas futuras de esta niña de 16 años.

Esta diferencia entre el castigo físico según el género, podría explicar, en algunos casos, cómo cada uno de ellos vive esta experiencia de adulto. El varón puede convertirse en un maltratador y la mujer en una víctima, cada uno respondiendo al significado que tuvo el maltrato para ellos. Desde esta última hipótesis, vamos a reflexionar sobre el maltrato.

\section{Propuesta teórica}

Proponemos para explicar el maltrato, sobre todo de tipo sexual, el trabajo de Ferenczi (1984) quien sostiene que los niños de diferentes clases sociales han sido violentados sexualmente a veces por sus propios padres, quienes buscan un sustituto a sus insatisfacciones de forma patológica, o pueden ser personas cercanas y de confianza a la familia, como los familiares, educadores o empleadas domésticas que pueden abusar de los niños.

Ferenczi (1984) sostiene que las llamadas seducciones incestuosas se dan cuando un adulto y un niño se aman, para el niño puede ser un juego, como tener un rol maternal en relación con adulto. Si bien, puede tener características eróticas, para el niño siempre se mantendrá en el ámbito de la ternura. Esto no pasa con los adultos, que presentan alguna psicopatología de su afectividad o son consumidores de drogas. Estos adultos confunden los juegos de los niños, los consideran deseos sexuales de un adulto maduro y tienen comportamientos sexuales sin tener en cuenta las consecuencias. Esto conduce a frecuentes violaciones sexuales de infantes o actos homosexuales impuestos. 
Para Ferenczi(1984), los niños se encuentran en desventaja, tienen temores y física y moralmente se encuentran indefensos. Psicológicamente se sienten inferiores ante la autoridad del adulto, quedan mudos, sin consciencia, dice Ferenczi; sumisos, dominados ante el agresor, ante su voluntad. Por lo tanto, obedecen y se olvidan de ellos mismos. Se identifican con el agresor, es decir se ha producido una introyección del agresor, el cual desaparece como realidad externa y se hace intrapsíquico y desde allí se puede expresar en diversas formas a través del principio del placer.

Lo que sería, para Ferenczi (1984), más significativo de esta identificación es la introyección del sentimiento de culpa del adulto, por lo que ahora este acto merece ser castigado. Por lo tanto, el niño se encuentra dividido entre la inocencia y la culpabilidad. El niño abusado obedece sin pensar, se vuelve obstinado, sexualmente inmaduro o perverso.

Por lo expuesto, Ferenczi i(1984) ndica que lo importante en el caso del maltrato o abuso es entender que este sujeto en desarrollo, reacciona al maltrato no defendiéndose, sino con una identificación ansiosa y la introyección de lo que lo amenaza o le agrede.

Ferenczi nos ayuda a entender este proceso, profundizando en el concepto de identificación, señalando que ya Freud, había sostenido que para que el niño pueda amar a otro tiene que ocurrir previamente, una identificación. Ferenczi denomina a este momento como un "estadio de la ternura". Donde los niños se imaginan y juegan a asumir el lugar del padre del mismo sexo para convertirse en la pareja del otro. Es claro, que los niños no pasan de este estadio de la ternura maternal a otro tipo de afecto. Por lo tanto, señala Ferenczi que si el niño es expuesto a un amor diferente o inclusive mayor al que desea, al de la ternura, este ocasiona un daño en la mente del niño tan similar o de mayor magnitud a cuando el niño es privado de afecto, de atención. Estas formas de "amor" que son apasionadas y llenas de culpabilidad en un niño en desarrollo e inocente dejan huellas patógenas en las diversas expresiones de la afectividad del niño.

Otra perspectiva señalada por Genoves (2019) quien cita a Ferenczi, nos ayuda a explicar otro concepto importante para comprender el maltrato. Este es denominado «terrorismo del sufrimiento». Hace referencia al comportamiento ejercido por las madres que se lamentan continuamente de sus sufrimientos y con ello convierten a su hijo en una ayuda cuidadosa y en un sustituto maternal, donde sus propios intereses son subordinados a los de la madre. Esto puede llevar al niño a ser un individuo que pueda colocar los intereses de los demás por encima de los personales y de esta forma puede permitir cualquier tipo de maltrato o abuso. 
Reflexionemos ahora sobre las consecuencias del maltrato o abuso sexual. Se sabe que el perjuicio del abuso se convierte en más dañino a nivel psicológico. Si bien Ferenczi ya lo había indicado. Es importante hacer hincapié en algunas consecuencias de este maltrato. Como sostiene Galán (2009) lo que ocurre en la mente del niño es lo siguiente: "Como mecanismo de defensa el niño buscará una identificación con el adulto, y esto le llevará a incorporar los sentimientos de culpa que corresponden al abusador. De esta manera, será un niño herido y, paradójicamente, culpable. Esto queda muy bien reflejado en dos visiones del concepto de «identificación con el agresor»”. Como indica Frankel (2002) citado por Galán (2009) "Este puede ser entendido en el sentido de Anna Freud, como un movimiento psíquico por el que se asumen los atributos del agresor, de modo que el amenazado se transforma en la persona que amenaza (Freud, 1971)". Esto implicaría que el niño maltratado se convierte, por un lado, en un maltratador, en un agresor, y comienza por ejemplo, en la escuela, en la adolescencia y luego en la adultez, con su pareja o relaciones interpersonales en general a dar muestras de maltrato.

Por otro lado, como explica Ferenczi, la identificación con el agresor, lleva a que el niño agredido, abusado, realiza un movimiento mental contrario al que suponía Anna Freud; es decir, "Ante una amenaza ineludible, para sobrevivir nos convertimos en lo que el atacante espera de nosotros; estamos por tanto ante una renuncia a nuestra identidad y el resultado será el opuesto al descrito por Anna Freud. Estaremos ante una acomodación y sumisión. Este planteamiento, junto con su idea de que el trauma implica una división en la personalidad (Ferenczi, 19), permite abrir la puerta a la comprensión de muchas características de los niños maltratados: la sumisión al agresor, los procesos disociativos, el sentimiento de culpa, la exposición futura a nuevas experiencias traumáticas, etc.”.

Daurella (2017), citando a Ferenczi, señala algunas características de la identificación con el agresor y que las colocaremos aquí porque amplían y aclaran este concepto clave en la psicogénesis del maltrato.

Daurella sostiene: "Pero Ferenczi da un alcance más amplio al mecanismo, describiendo tres acciones virtualmente simultáneas:

a) El niño se somete mentalmente al agresor.

b) Esta sumisión le permite adivinar los deseos del agresor. Podríamos decir (y esto no lo dice Ferenczi, sino que es cosecha propia) que se produce una auténtica "criptestesia" (es decir, un 
estado de la sensibilidad más aguzada de lo normal que se encuentra en la base de fenómenos como las premoniciones y las adivinaciones.

c) El niño hace una especie de pseudo-progresión traumática o pre-maduración patológica que le permite hacer aquello que él siente que le salvará.

Profundizando en la comprensión de todo tipo de maltrato, es importante recalcar que el mecanismo de defensa denominado "identificación con el agresor", se lo describe así: "personificando el agresor, asumiendo sus atributos o imitando su agresión, el niño se transforma de ser amenazado, a ser quien profiere la amenaza". Y esto lo podemos observar en el comportamiento de los varones que desde niños son pegadores y en especial con los más débiles, por ejemplo las mujeres.

Examinemos ahora la identificación con el agresor en el caso de las niñas. Se puede referir entonces a un factor de género, de condicionamiento de rol. Tendríamos que encontrar otra explicación en el concepto planteado por Ferenczi, que es citado también por Abad (2009):

El proceso que describe Ferenczi, y que se produce de forma inconsciente, es el siguiente:

1. El niño/a abusado se somete.

2. El sometimiento permite estar vigilante, atento y llegar así a poder adivinar los deseos del otro: Penetrar de esa manera, en la mente del atacante y poder "ver" qué está sintiendo para poder anticipar el próximo golpe y estar preparado.

3. En la medida que pueda anticipar y prepararse, minimiza los efectos no deseados.

4. Por lo indicado en el punto 2, los sentimientos propios llegan a desconocerse, y se da una falta de contacto con la propia subjetividad, por eso no hay rabia, sino un vaciamiento creciente de la subjetividad.

Abad explica esta identificación con el agresor en el caso de las mujeres. Sostiene lo siguiente: "Esto es lo mismo que la feminidad estereotipada. Freud se preguntaba qué desean las mujeres, porque hay un cierto vaciamiento "normalizado" de lo que tiene que ser una "buena mujer". El contínuum entre condiciones patológicas, y condiciones patológicas muy normalizadas socialmente da lugar a mujeres estereotipadas". Lo descrito podemos observarlo en las noticias sobre violencia contra la mujer, donde está normalizado, que ser una buena mujer implica dejarse golpear y donde la niña, ahora mujer que ha sido abusada se somete a su agresor. Eso 
explica, cómo en algunos casos, luego de tanta violencia, los sentimientos propios han dejado de ser considerados y solo lo importante es lo que el atacante desee, lo que él quiera.

Por otro lado con relación al maltrato de las mujeres, se sabe que los varones abusados o maltratados, cuando están con su pareja, se toman la revancha, pero no contra sí mismos, sino contra la pareja. Hay una transmisión generacional de las condiciones de abuso y de maltrato.

Frankel (2002) citado por Abad (2009) explica que lo más dañino del abuso en la infancia es la dominación y clausura de la mente del niño. Esto sucede "cuando se depende física y emocionalmente de otro que viola y explota, cuando... una persona tiene concedida la autoridad de controlar y definir la realidad del otro, incluso cuando la definición de esa realidad subsista en duro contraste con la experiencia real vivida por la persona”. Por ello, otro de los trabajos a realizar en salud emocional, con los jóvenes, es la prevención de la dependencia emocional del otro. Esto es nuclear en la formación de las futuras parejas, pero claro, más importante será prevenir y evitar el abuso infantil en todas sus formas.

Otro concepto clave en el maltrato es del sentimiento de culpa. Como sabemos, Ferenczi señala que "el aspecto más dañino de la identificación con el agresor es lo que llamó la "introyección de los sentimientos de culpa del adulto". El niño víctima de abuso se echa la culpa a sí mismo por lo sucedido y se siente malo. Este niño se ha identificado con la maldad del abusador y probablemente con la percepción del abusador de que el niño es malo. El término de Ferenczi implica que todos los abusadores sienten culpa, lo cual no es cierto. Pero la introyección está ciertamente involucrada cuando un niño asume la maldad de su agresor, porque ese niño internaliza y reorganiza los hechos abusivos reales en su mente para convertirse a sí mismo en el causante de su propio abuso. Este sentimiento grandioso de control es preferible a encarar la realidad de ser una víctima desamparada". Esto implica la necesidad de enfrentar cualquier situación de revelación de un niño que ha sido abusado, trabajando la culpa, que no lo lleva a sentirse víctima, sino responsable de lo que le pasó porque él es malo. Esto es clave para entender por qué muchos niños abusados, no hablan, no denuncian, por sentirse desamparados, piensan que nadie los entenderá y perdonará. La desculpabilización de los niños, que de adultos llegan a consulta, debe ser uno de los primeros objetivos psicoterapéuticos a trabajar con estos pacientes.

Otro aporte importante de Abad (2009) sobre el abuso sexual y su origen se refiere a la necesidad de diferenciar las edades de los niños para hablar de abuso. En este sentido los juegos sexuales de niños de la misma edad no pueden ser considerados abusos. La diferencia de edad 
debe ser el doble, por ejemplo un niño de 12 con uno de 5 o 6 años si podemos considerar como abuso.

Otra diferenciación importante es el abuso extrafamiliar y el intrafamiliar. En el primero, cuando ocurre, el niño se siente víctima y no se altera su identidad, no tiene que disociarse, no se siente culpable. No deja de ser grave el abuso extrafamiliar, pero indudablemente el intrafamiliar es más grave en el tiempo y en las huellas mnémicas que deja en el inconsciente.

En la modalidad intrafamiliar, como señala Adad, el niño se siente culpable, porque piensa que es él o ella, que provocó o incitó al abusador y también por quedarse callado o no pedir ayuda. Así mismo, en la modalidad intrafamiliar se produce una disociación, en la cual hay una percepción de algo que se da y no se da, porque el niño se va mentalmente del hecho en el momento que está ocurriendo el abuso. Así mismo Abad indica que lo que se da en ese instante, se puede denominar una "dislocación del sentido" porque el niño confunde los roles y las reglas. Por ejemplo se altera la relación adulto y niño, de lo que implica la protección y lo que no lo es, así como lo que implica el incesto. Hay por lo tanto una alteración de la realidad, que tiene que ver con el juicio, con lo mental y que por lo tanto no es solamente una alteración de la memoria.

Finalmente presentaremos la propuesta de Diaz-Benjumea (2011) sobre los factores causales del abuso. Ella plantea los siguientes:

1. Ser del género femenino: es un riesgo ser mujer cuando nos referimos a la violencia de género. Diaz-Benjumea cita a Bleichmar $(1995,1997)$ y a Levinton (2000) para hacer referencia a los ideales de género, como aquello que se espera del hombre y de la mujer en una sociedad. En este sentido señala, que la subjetividad femenina está constituida por todas las introyecciones de los ideales que su medio le brinda a cada mujer.

Estos mandatos y pautas sociales tienen que ver con expectativas relacionadas a los afectos y a las emociones, a la capacidad de darse cuenta y atender las necesidades de los otros y se alejan de los espacios para expresar la agresión por ejemplo, que es considerada contraria a lo femenino y a la maternidad. La mujer debe ser dócil, se la considera en cuanto a su sexualidad como un objeto de deseo y se la denigra si ella se considera como sujeto de deseo.

Por otro lado, a la mujer se le atribuye el ser vulnerable y dependiente; mientras que el hombre es autónomo e independiente, la mujer es frágil, no puede hacer sola sus cosas y no puede con 
todo, por ello depende del hombre. Este tipo de identidad, tal como lo señala Diaz-Benjumea está estructurada con base en la debilidad, a base de la precariedad, es una identidad incompleta que solo puede completarse en la relación heterosexual con un hombre. Es una identidad, dirá la autora, de minusvalía.

Definitivamente, la mujer asume un rol que la desfavorece, que la limita frente al hombre y la sociedad y que promueve por lo tanto, la posibilidad de pasar por encima de ella, incluso maltratarla y abusar de ella, sin considerar que ello sea algo anormal, sino que responde a los ideales del género que le hemos atribuido como sociedad a la mujer.

2. Historia de abuso en la infancia: Diaz-Benjumea sostiene que se produce un trauma en las niñas que han sufrido abuso sexual infantil sobre todo a nivel intrafamiliar. Señala los estudios de Walker (2009) que realiza un trabajo con las mujeres que quedan atrapadas en relaciones de violencia de género. Dependiendo del tipo de maltrato, realizado por un miembro de la familia, en un tiempo prolongado deja secuelas a nivel intrasubjetivo y también intersubjetivos, donde estas mujeres tienden a involucrarse con hombres que las maltratan tanto física como psicológicamente, tal como lo demuestras los estudios de Bateman y Fonagy (2007) y Davis y Frawlay (1994) citados por Diaz-Benjumea en el presente estudio.

3. Una historia de apego no seguro: Diaz-Benjumea se refiere ahora a las experiencias o vivencias relacionadas con lo cotidiano y a los vínculos con los cuidadores. En este sentido, los cuidadores no han desempeñado este rol, no han brindado seguridad, protección, confianza básica. Mas bien se han producido experiencias de abandono, de descuido, de no valoración. Donde los cuidadores han estado presentes solo en algunos momentos de la vida y del desarrollo de la niña. Su disposición al contacto físico y psicológico han sido ocasional. Ello ha creado en la niña un apego inseguro, un apego ansioso. La niña buscará en el futuro un apego seguro, este vínculo que le faltó en su desarrollo temprano. Por lo tanto, esta niña no sabrá regular su ansiedad, porque no lo ha vivenciado. No ha interiorizado objetos internos buenos que lo ayuden a sobrellevar la soledad existencial, no ha logrado sentirse reconocida como sujeto de valía, como persona que necesita ser comprendida por el otro. Desde esta perspectiva Diaz-Benjumea cita a Goldner(2004) quien en sus estudios encuentra una relación entre la falta de reconocimiento de las mujeres que son víctimas de violencia. Esto explica que estas mujeres acepten cualquier tipo de atención, aunque sea violenta, con tal de sentirse reconocidas, algo que por ejemplo, una madre que busca más bien el afecto y ser emocionalmente calmada por su hija y un padre que evade los problemas y es periférico en su 
relación, no lograron conseguir. Este es el problema de no haber desarrollado un apego seguro en las niñas.

4. La vivencia de la violencia en la pareja: Diaz-Benjumea señala que esta vivencia es por sí traumática y lo peor de ella es que deja a la mujer sin medios para defenderse o escapar de dicha situación. Los estudios señalan que la instauración de la violencia es por ciclos, donde se han pasado de situaciones de conflicto en la pareja a momentos de calma, que han hecho pensar que todo podía mejorar. Se han tomado en cuenta en este tipo de causa del maltrato las múltiples razones para no defenderse y salir de este círculo dañino. Entre ellas tenemos el miedo, la baja autoestima, la indefensión, lo imprevisible de la agresión, el aislarse de otros seres queridos. A todo esto se suman las creencias relacionadas con los ideales de género sobre el rol de la mujer de ser afectuosa y la concepción de un amor romántico idealizado. Todo esto contribuye para que poco apoco la mujer vaya perdiendo los recursos para defenderse que en otras situaciones se activarían normalmente.

De este modo, en la comprensión del maltrato, podemos encontrar, por un lado la creación de un perpetrador, abusador, maltratador y por el otro se construye una víctima que acepta ser dominada por el otro, que acepta con sumisión todo lo que venga del maltratador y que nos llevaría a entender por qué muchas mujeres golpeadas son encontradas al día siguiente nuevamente en el lecho con sus parejas y cuando la policía quiere detenerlos, inclusive los defienden. Se reactivan en ellas y ellos este miedo a este adulto que ha sido todopoderoso, y al final se somete a él.

\section{A modo de conclusiones:}

No podemos entender el maltrato, sin tener en cuenta las propuestas estudiadas en el artículo, sin entender la identificación con el agresor, sin estudiar el desarrollo de los niños y niñas y de sus cuidadores, sin cuestionar los ideales de género que la sociedad demanda a la mujer, sin educar a los futuros padres de familia, sin encontrar que la búsqueda de la prevención de la violencia es social, estructural; pero también es familiar y sobre todo entender que una mirada profunda de nuestras historias personales puede hacer el cambio y pasar de maltratados y víctimas, a protectores, a tutores de resiliencia, a psicoterapeutas de niños y adolescentes, a educadores garantes de los derechos de los niños y jóvenes. 
Es claro que el maltrato ocurre durante el desarrollo de los niños y niñas. Traumatiza la misma experiencia de abuso. El hecho en sí. Pero hoy sabemos, que también traumatiza el haber vivido con cuidadores que no cumplieron con su rol de protección. También sabemos que puede no haber ocurrido el hecho de maltrato en sí. Pero basta que hayamos estado en un ambiente que nos haya infundido el miedo o el temor de que el maltrato podría ocurrir para entender que existan adultos que se conviertan en víctimas o victimarios.

Hemos encontrado que los niños golpeados no solo pueden identificarse con los agresores y los imitan, sino que también pueden perder su identidad, y convertirse por el maltrato en personas sumisas y dominadas, donde lo más importante no es el propio interés sino lo que el abusador desea.

La dependencia emocional se forma tempranamente. Para que no se dé, necesitamos padres que brinden un apego seguro y para ello deben estar dispuestos a vincularse la mayor parte del tiempo con las necesidades afectivas de sus hijos, no solo cuando se pueda u ocasionalmente. Tampoco ayuda el abandono porque crea la inseguridad y la no introyección de objetos internos que ayuden a enfrentar la vida y las ansiedades propias de la existencia humana.

Es necesario, cuidadores afectivamente maduros, que no depositen en sus hijos sus carencias o dificultades, que no los utilicen para sostenimiento emocional, porque esto logrará una confusión de roles, entre padres e hijos que no ayuda a sentirse seguros y protegidos cuando es el tiempo del desarrollo para estarlo.

El psicoanálisis nos explica entonces que no es casual, lo que origina el maltrato, sino que existe el registro de todas nuestras vivencias, registro de nuestras experiencias, pero también de nuestras ideas, de nuestras emociones, y es este registro el que utilizamos para comportarnos. Registro que nos ayuda a entender como se ha instaurado en nuestra psique las bases para ser maltratados, para ser víctimas, para ser abusadores y que son registros que quedan como huellas mnémicas en la memoria biológica inconsciente y que se reactivan en situaciones de violencia o entornos desfavorables. Toda persona que ha sido maltratada o abusada, de no ser tratada, atendida desde la psicología, desde la psicoterapia, desde la educación, desde todos los sistemas sociales y redes de protección, perpetuará el ciclo de violencia y anomia social que ahora vivimos. Hará más difícil alejarnos y prevenir el maltrato en nuestra sociedad. 
Por lo tanto, la responsabilidad es de todos, no podemos quedarnos simplemente atónitos ante las noticias de violencia, pensar que son normales y parte de nuestra sociedad. Tenemos que cuestionarla, buscando explicaciones y soluciones. Al concluir este artículo, aún no dejo de pensar en la última noticia que informó de la violación a una joven de 22 años por parte de cinco de sus "mejores amigos". No podemos quedarnos indiferentes o pensar, es una noticia más y seguir adelante con nuestras vidas. No podemos avalar la justificación de uno de los abogados de los victimarios, que dijo a la prensa "ella era una chica movida, que le gustaba la diversión”. Sería normalizar estos ideales de género que hemos estudiado en el artículo, donde se describe cómo debe ser el comportamiento de una mujer. Nos enfrentamos a una tarea más compleja y estructural, pero tenemos que recurrir a todas las especialidades y estudios para lograr un cambio desde lo más profundo. Todos estamos llamados a ser parte de la solución. Por ahora, procuremos cumplir nuestro rol, lo mejor posible, ser buenos cuidadores, protectores, creadores de apego seguro con nuestros niños, cercanos, dispuestos, afectivamente maduros. Esos adultos necesitan nuestros niños, adultos sanos emocionalmente, dispuestos a cuidar a proteger, a ofrecer entornos seguros y favorables para el desarrollo y bienestar de los niños.

\section{Referencias}

Abad, M. (2009). El abuso sexual. Comentario sobre un caso clínico. En: Aperturas Psicoanalíticas. Revista de Psicoanálisis en Internet. Número 031.

\section{Cyrulnik, B. (2010). El amor que nos cura. Barcelona. Editorial Gedisa}

Diaz-Benjumea, L. (2011). Mecanismos psíquicos implicados en la tolerancia de las mujeres al maltrato. Un enfoque de subtipos de mujeres maltratadas. En: Revista Internacional Psicoanálisis on-line

Ferenczi, S. (1984). Psicoanális. Tomo IV. Obras Completas. Madrid. Editorial Espasa-Calpe.

Frankley, J.(2002). Explorando el concepto de Ferenczi de identificación con el agresor. Su rol en el trauma, la vida cotidiana y la relación terapéutica. En: Revista Internacional de Psicoanálisis Aperturas. № 011

Galán, A. (2009). Psicoanálisis y la protección a la infancia. Recuperado de: 
https://www.fundacioorienta.com/wp-content/uploads/2019/02/Galan-Antonio-15.pdf

Genovés, A. (2019). Ferenczi, S. (1984). Confusión de lenguas entre los adultos y el niño. El lenguaje de la ternura y de la pasión En: Aperturas Psicoanalíticas, Recuperado de: http://aperturas.org/articulo.php?articulo=0001087

OMS (2020). Global status report on preventing violence against children 2020. Recuperado de:

https://www.who.int/teams/social-determinants-of-health/violence-prevention/global-statusreport-on-violence-against-children-2020 